\title{
Secrecy Energy-Efficient UAV Communication via Trajectory Design and Power Control
}

\author{
An Li $\mathbb{D}^{\text {, }}$, Huohuo Han $(\mathbb{D}$, and Chuanxin Yu \\ Department of Electronic Information Engineering, Nanchang University, Nanchang 330031, China \\ Correspondence should be addressed to An Li; lian@ncu.edu.cn
}

Received 28 March 2021; Revised 24 July 2021; Accepted 28 August 2021; Published 17 September 2021

Academic Editor: Stefan Panic

Copyright ( 2021 An Li et al. This is an open access article distributed under the Creative Commons Attribution License, which permits unrestricted use, distribution, and reproduction in any medium, provided the original work is properly cited.

\begin{abstract}
This paper investigates the problem of maximizing the secrecy energy efficiency (SEE) for unmanned aerial vehicle- (UAV-) toground wireless communication system, in which a fixed-wing UAV tries to transmit covert information to a terrestrial legitimate destination receiver with multiple terrestrial eavesdroppers. In particular, we intend to maximize the worst-case SEE of UAV by jointly optimizing UAV's flight trajectory and transmit power over a finite flight period. However, the formulated problem is challenging to solve because of its large-scale nonconvexity. For efficiently solving this problem, we first decouple the above optimization problem into two subproblems and then propose an alternating iterative algorithm by adopting block coordinate descent method and Dinkelbach's algorithm as well as successive convex approximation technique to seek a suboptimal solution. For the sake of performance comparison, two benchmark schemes, the secrecy rate maximization (SRM) scheme and constrained energy minimization (CEM) scheme are considered to obtain more useful insights. Finally, simulation results are executed to verify that our proposed SEE maximization (SEEM) algorithm is superior to two benchmark schemes for the UAV-ground communication system.
\end{abstract}

\section{Introduction}

According to Global Information, Inc. (GII) [1], by 2027, the UAV market will reach $\$ 21.8$ billion and 13.2 million vehicles in terms of volume. Particularly, UAVs have been viewed as an appealing technique to aid ground communication due to their swift for deployment, low cost, flexible mobility, and adaptive altitude [2]. For example, there has been considerable work on UAVs operating as airborne communication platforms, such as stationary/mobile aerial cellular base stations (BSs)/access points (APs) [3, 4], mobile relays [5], mobile data collectors [6], mobile cooperative jammer [7], and mobile edge computing servers [8]. However, although UAVs usually have line-of-sight (LoS) links for air-to-ground communications to improve the received signal level, such LoS links become also more susceptible to interception by terrestrial eavesdroppers (Eves), thus leading to a new security threat. Physical layer security (PLS) has become an effective approach to enable secure wireless communication, where the rich physical layer features and resources of the wireless channel can be effectively exploited to enhance the security of the wireless link and achieve secure transmission.

Over the past few years, there are plenty of PLS schemes proposed to enable secure terrestrial wireless communication. Specifically, they can be classified in two main categories: first, schemes based on introducing multiple-level cooperative relay [9] or multiple-input multiple-output (MIMO) [10] to improve the legitimate channel capacity and second, noise forward- [11] or cooperative jammingbased $[12,13]$ schemes to significantly reduce the eavesdropping channel capacity. However, only fixed or quasistatic ground nodes are considered in the above existing PLS research, thus bringing the following two challenges. First, when the fixed distance between the source and destination node is far larger than that between the source and a potential eavesdropping node, the existing physical layer security transmission methods only provide the extremely limited security performance improvement for the wireless system. Second, PLS schemes based on transmit power control, beamforming, and/or cooperative jamming usually require precisely known channel state information (CSI) of 
eavesdropper. However, the terrestrial wireless channel is rather random, especially for the case in the presence of the passive eavesdroppers, which makes CSI estimation very difficult.

Thanks to the controllable mobility and LoS channel of UAV, the use of UAV is expected to solve the above challenges. On the one hand, UAVs as airborne communication platforms can move as close to (far away from) the destination (eavesdropper) as possible, thus improving (degrading) the channels from the UAV to destination (eavesdropper). On the other hand, the CSI between UAV and terrestrial nodes including eavesdroppers can be easily obtained due to the LoS link. Note that the location of eavesdropper is easy to obtain via UAV-based radar or camera [14].

1.1. Related Works. Recently, a number of studies have paid attention to improve the secrecy performance of UAV communication via applying PLS [7, 15-19]. Specifically, the authors in [15] first investigated the issue of PLS for a downlink UAV communication system against the terrestrial eavesdropper, and a novel design paradigm is proposed to achieve maximum average secrecy rate via jointly designing the UAV transmit power and trajectory. In [7], the authors first presented to utilize a UAVenabled mobile jammer to improve the system achievable secrecy rate, which provide new insights to UAV applications. The works [16-18] further considered two UAVs to secure the communication system, where one UAV sends confidential information and the other acts as a friendly mobile jammer to send jamming signal to interfere ground eavesdropper to maximize secrecy rate. In addition, a more practical scenario for UAV-ground communication, where two UAVs perfectly know the information on the location of legitimate ground node while the ground eavesdropper's location is not perfectly known, was investigated in [19]. The worst-case secrecy rate of the UAV communication network is maximized by jointly optimizing the UAVs' trajectories and their communicating/jamming power allocations. However, the aforementioned works just addressed the SRM optimization problem via a proper UAV trajectory design without taking the power consumption into account.

In fact, for the UAV-ground communication system, such controllable mobility usually consumes more propulsion energy (far greater than the energy related to communication), which is mainly supplied by batteries. However, a UAV's on-board energy is another major problem because of the size and weight limitations of UAV, which severely limits the sustainability and performance of UAV communication systems. Following the first work of Zeng and Zhang [20] on the analytical UAV propulsion energy consumption model, researchers have widely developed a few energyefficient UAV communication schemes to strike an optimal balance between the system throughput and the energy consumption and further prolong the UAV endurance [21-23]. Nevertheless, all these works have not considered the security issue for UAV communication, which motivates us to investigate how to jointly provide secrecy enhancement and save UAV's energy.
Although there has been a little work on the study of energy efficiency (EE) of UAV secrecy communications, such as $[24,25]$, the authors in [24] investigated the secrecy energy efficiency (SEE) maximization of a UAV-aided dualhop transmission system with a potential eavesdropper by jointly designing the UAV transmit power, flight trajectory, and communication scheduling. In [25], a full duplex $\mathrm{UAV}$-aided secure communication scheme was proposed, in which the UAV collects the confidential messages from a source node on the ground and, at the same time, sends jamming signals to interfere eavesdropping. The system SEE is maximized via optimizing the UAV's jamming power and flight trajectory, jointly with source transmit power allocation. However, $[24,25]$ focused on the SEE optimization for UAV-assisted relaying or data gathering with a single eavesdropper, respectively. To the best of our knowledge, there is no existing work to study the issue of maximizing the worst-case SEE for the UAV-ground communication system with multiple terrestrial eavesdroppers.

1.2. Contributions. Motivated by the above works, we jointly consider the UAV's energy consumption and the system secrecy rate for a UAV-ground communication system with multiple ground eavesdroppers in this paper. Specifically, a fixed-wing UAV is exploited to transmit confidential information to terrestrial destination, and the worst-case SEE is maximized via optimizing UAV trajectory, flight velocity and acceleration, and transmit power.

Here are the main contributions of this paper:

(i) To strike an optimal balance between the UAV's energy consumption and the system secrecy rate, we propose a joint UAV trajectory design and power allocation scheme to maximize the system worst-case SEE

(ii) The formulated SEE maximization problem is challenging to solve because of its large-scale nonconvexity. To handle this intractable issue, we present an iterative algorithm to find the high-quality local optimum via employing the block coordinate descent (BCD) method and Dinkelbach's algorithm as well as successive convex approximation (SCA) technique

(iii) To obtain more performance insights, the SEE for the secrecy rate maximization (SRM) scheme and constrained energy minimization (CEM) scheme are also considered for comparison. Numerical results manifest that our proposed scheme can lead to a significant improvement on system SEE over the benchmark schemes

\section{System Model and Problem Formulation}

2.1. System Model. We consider a secure UAV-ground wireless transmission system, as shown in Figure 1, where a fixed-wing UAV is deployed to secretly transmit information to a terrestrial destination (Bob) in the existence of $K$ terrestrial eavesdroppers (Eves). Considering a three- 


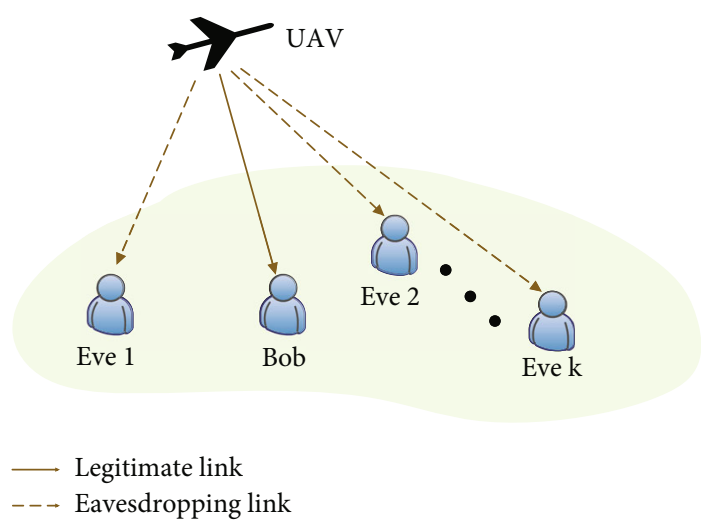

Figure 1: A secure UAV-ground communication system with multiple eavesdroppers.

dimensional Cartesian coordinate system, we assume that the nodes Bob and Eves are in the fixed locations on the ground, denoted by $\mathbf{q}_{\mathrm{B}}=[0,0]^{T}$ and $\mathbf{E}_{k}=\left[x_{k}, y_{k}\right]^{T}, k \in \mathscr{K} \triangleq$ $\{1, \cdots, K\}$, respectively. Note that this location information is perfectly known to UAV. Let $\mathbf{q}(t)=[x(t), y(t)]^{T}$ represent the UAV trajectory projected coordinate on the ground at time instant $t \in[0, T]$, where $T$ in seconds (s) is the UAV's serving period. Therefore, the corresponding velocity and acceleration of UAV at $t$ are, respectively, denoted by $\mathbf{v}(t)$ $=\dot{\mathbf{q}}(t)$ and $\mathbf{a}(t)=\ddot{\mathbf{q}}(t)$.

We assume that the UAV aviates horizontally on a constant altitude $H$ meters $(\mathrm{m})$ within a finite flight period $T$ from a start point $\mathbf{q}_{0}$ to end point $\mathbf{q}_{\mathrm{F}}$, which are both predetermind by the mission/task. For simplicity, we discretize the period $T$ into $N$ time slots, i.e., $T=N \delta_{t}$, where the equalspaced time interval $\delta_{t}$ is chosen small enough such that during each time slot the UAV's location can be roughly considered to be constant. Consequently, the horizontal trajectory of UAV $\mathbf{q}(t)$ over flight period $T$ can be approximately represented by $N$-length sequence $\mathbf{q}[n]=[x[n], y[n]]^{T}, n \in \mathcal{N}=\{0,1, \cdots, N\}$. Following [21], the UAV satisfies the following mobility constraints:

$$
\begin{gathered}
\mathbf{q}[0]=\mathbf{q}_{0}, \mathbf{q}[N+1]=\mathbf{q}_{\mathrm{F}}, \\
\mathbf{q}[n+1]=\mathbf{q}[n]+\mathbf{v}[n] \delta_{t}+\frac{1}{2} \mathbf{a}[n] \delta_{t}^{2}, n \in \mathcal{N}, \\
\mathbf{v}[0]=\mathbf{v}_{0}, \mathbf{v}[N+1]=\mathbf{v}_{\mathrm{F}}, \\
\mathbf{v}[n+1]=\mathbf{v}[n]+\mathbf{a}[n] \delta_{t}, n \in \mathcal{N}, \\
\|\mathbf{v}[n]\| \leq V_{\max }, n \in \mathcal{N}, \\
\|\mathbf{v}[n]\| \geq V_{\min }, n \in \mathcal{N}, \\
\|\mathbf{a}[n]\| \leq a_{\max }, n \in \mathcal{N},
\end{gathered}
$$

where $\mathbf{v}[n]$ and $\mathbf{a}[n]$ are the UAV's velocity and acceleration, respectively; $\mathbf{v}_{0}$ and $\mathbf{v}_{\mathrm{F}}$ are the given initial and final velocity, respectively; $V_{\max }$ and $V_{\min }$ are the maximum and minimum speeds in each slot $n$, respectively; and $a_{\max }$ represents the maximum acceleration. Thus, the distance within slot $n$ from UAV to the Bob or Eve $k$ can be expressed as

$$
\begin{gathered}
d_{\mathrm{UB}}[n]=\sqrt{H^{2}+\|\mathbf{q}[n]\|^{2}}, \\
d_{\mathrm{UE}_{k}}[n]=\sqrt{H^{2}+\left\|\mathbf{q}[n]-\mathbf{E}_{k}\right\|^{2}} .
\end{gathered}
$$

Assuming that the UAV-ground channel is dominated by LoS links [26], and the Doppler effect due to UAV mobility can be completely compensated by the receiver simultaneously. Therefore, for each slot $n$, the channel power gain from UAV to ground nodes is given by

$$
\begin{array}{r}
h_{\mathrm{UB}}[n]=\beta_{0} d_{\mathrm{UB}}^{-2}[n]=\frac{\beta_{0}}{H^{2}+\|\mathbf{q}[n]\|^{2}}, \\
h_{\mathrm{UE}_{k}}[n]=\beta_{0} d_{\mathrm{UE}_{k}}^{-2}[n]=\frac{\beta_{0}}{H^{2}+\left\|\mathbf{q}[n]-\mathbf{E}_{k}\right\|^{2}},
\end{array}
$$

where $\beta_{0}$ denotes the channel power gain at the reference distance $d_{0}=1 \mathrm{~m}$.

In each time slot $n$, let $P[n]$ represent the transmit power of UAV, which is subjected to both average and peak power constraints, represented as $\bar{P}$ and $P_{\text {peak }}$, respectively, i.e.,

$$
\begin{aligned}
& \frac{1}{N} \sum_{n=1}^{N} P[n] \leq \bar{P}, n \in \mathcal{N}, \\
& 0 \leq P[n] \leq P_{\text {peak }}, n \in \mathcal{N} .
\end{aligned}
$$

Let $\bar{P}<P_{\text {peak }}$ in this paper to make constraints (4a) nontrivial. Hence, the maximum achievable rate between Bob and UAV in time slot $n$ in bits/second is given by [27].

$R_{\mathrm{UB}}[n]=B \log _{2}\left(1+\frac{p[n] h_{\mathrm{UB}}[n]}{\sigma^{2}}\right)=B \log _{2}\left(1+\frac{\gamma_{0} P[n]}{H^{2}+\|\mathbf{q}[n]\|^{2}}\right)$,

where $B$ is the communication bandwidth, $\sigma^{2}$ is the noise power at the receiver, and $\gamma_{0}=\beta_{0} / \sigma^{2}$. Similarly, the achievable rate of Eve $k$ in bits/second at time slot $n$ is calculated as

$$
R_{\mathrm{UE}_{k}}[n]=B \log _{2}\left(1+\frac{\gamma_{0} P[n]}{H^{2}+\left\|\mathbf{q}[n]-\mathbf{E}_{k}\right\|^{2}}\right) .
$$

With (5) and (6), the average worst-case secrecy rate from UAV to Bob, in the existence of $K$ Eves, over the period $T$ in bits/second is given by [28]

$$
R_{\mathrm{sec}}=\frac{\delta_{t}}{N} \sum_{n=1}^{N}\left[R_{\mathrm{UB}}[n]-\max _{k \in \mathscr{K}} R_{\mathrm{UE}_{k}}[n]\right]^{+},
$$

where $[x]^{+} \triangleq \max (x, 0)$.

During the secure communication period $T$, the total energy consumption of UAV is mainly composed of the 
communication-related energy and the propulsion energy. The communication-related energy is ignored in our design since it is extremely small compared with the UAV's propulsion energy [20]. Therefore, our work focuses on the propulsion energy in Joule consumed over period $T$, which is upper bounded by according to [20]

$$
E(\{\mathbf{v}[n], \mathbf{a}[n]\})=\delta_{t} \sum_{n=1}^{N}\left(c_{1}\|\mathbf{v}[n]\|^{3}+\frac{c_{2}}{\|\mathbf{v}[n]\|}\left(1+\frac{\|\mathbf{a}[n]\|^{2}}{g^{2}}\right)\right)+\Delta_{k},
$$

where $g=9.8 \mathrm{~m} / \mathrm{s}^{2}$ represents the gravitational acceleration, $\Delta_{k}=(1 / 2) m\left(\left\|\mathbf{v}_{\mathrm{F}}\right\|^{2}-\left\|\mathbf{v}_{0}\right\|^{2}\right)$ denotes the kinetic energy change of the UAV, $m$ represents the mass of the UAV, and $c_{1}$ and $c_{2}$ are two constant parameters determined by aerodynamics forces.

With (7) and (8), the worst-case secrecy energy efficiency (SEE) in bits/J of the UAV-ground communication system in the existence of multiple eavesdroppers is written by

$$
\eta_{\mathrm{SEE}}=\frac{N R_{\mathrm{sec}}}{E(\{\mathbf{v}[n], \mathbf{a}[n]\})}
$$

2.2. Problem Formulation. For ease of exposition, let $\mathbf{q} \triangleq$ $[\mathbf{q}[0], \cdots, \mathbf{q}[N]]^{T}, \mathbf{v} \triangleq[\mathbf{v}[0], \cdots, \mathbf{v}[N]]^{T}, \mathbf{p} \triangleq[P[0], \cdots, P[N]]^{T}$, and $\mathbf{a} \triangleq[\mathbf{a}[1], \cdots, \mathbf{a}[N]]^{T}$. For secure UAV communication, we aim to maximize the worst-case SEE $\eta_{\text {SEE }}$ in (9) via jointly optimizing the UAV transmit power $\mathbf{p}$ and flight trajectory q under the constraints of transmit power in (4) as well as UAV mobility in $((1))$ within the service period $T$. To this end, the system achievable SEE maximization problem can be recast to

(P1):

$$
\begin{gathered}
\max _{\mathbf{p}, \mathbf{q}, \mathbf{v}, \mathbf{a}} \frac{\sum_{n=1}^{N}\left[R_{\mathrm{UB}}[n]-\max _{k \in \mathscr{K}} R_{\mathrm{UE}_{k}}[n]\right]}{\sum_{n=1}^{N}\left(c_{1}\|\mathbf{v}[n]\|^{3}+\left(c_{2} /\|\mathbf{v}[n]\|\right)\left(1+\left(\|\mathbf{a}[n]\|^{2} / g^{2}\right)\right)\right)+\left(\Delta_{k} / \delta_{t}\right)}, \\
\text { s.t. }(1),(4),
\end{gathered}
$$

where $[\cdot]^{+}$in (7) is omitted since the objective function in (10) must be nonnegative at the optimal solution; otherwise, without violating the transmit power constraints in (4), the value of $(\mathrm{P} 1)$ is at least zero by setting $P[n]=0$. Although the problem $(\mathrm{P} 1)$ is reformulated as a more tractable form, it is still nonconvex involving variables $\mathbf{p}, \mathbf{q}, \mathbf{v}$, and $\mathbf{a}$ and difficult to solve optimally. Therefore, we put forward an efficient iterative algorithm to address the problem (P1) in the following section.

\section{Proposed Solution}

In this section, an effective alternating iterative algorithm is proposed to deal with (P1) via applying block coordinate descent method and Dinkelbach's algorithm. Specifically, the problem (P1) is decomposed into two subproblems to separately optimize the UAV's transmit power $\mathbf{p}$ and the UAV's trajectory q. Two subproblems are alternatively optimized in an iterative manner till convergence.

3.1. Subproblem 1: Transmit Power p Optimization. Given any feasible UAV's trajectory $\mathbf{q},(\mathrm{P} 1)$ is simplified into the following form:

$$
\begin{gathered}
\max _{\mathbf{p}} \sum_{n=1}^{N}\left[\log _{2}\left(1+a_{n} P[n]\right)-\log _{2}\left(1+b_{n} P[n]\right)\right], \\
\text { s.t.(4), }
\end{gathered}
$$

where

$$
\begin{gathered}
a_{n}=\frac{\gamma_{0}}{H^{2}+\|\mathbf{q}[n]\|^{2}}, \\
b_{n}=\frac{\gamma_{0}}{H^{2}+\min _{k \in \mathscr{K}}\left\|\mathbf{q}[n]-\mathbf{E}_{k}\right\|^{2}} .
\end{gathered}
$$

Although problem (P2) is a nonconvex function of the UAV transmit power $\mathbf{p}$, its optimal solution can be obtained as shown in [28].

$$
P^{*}[n]=\left(\begin{array}{ll}
0, & a_{n} \leq b_{n}, \\
\min \left([P \wedge[n]]^{+}, P_{\text {peak }}\right), & a_{n}>b_{n},
\end{array}\right.
$$

where

$P \wedge[n]=\sqrt{\left(\frac{1}{2 b_{n}}-\frac{1}{2 a_{n}}\right)^{2}+\frac{1}{\lambda \ln 2}\left(\frac{1}{b_{n}}-\frac{1}{a_{n}}\right)}-\frac{1}{2 b_{n}}-\frac{1}{2 a_{n}}$,

where $\lambda$ should be a nonnegative parameter that ensures $\sum_{n=1}^{N} P^{*}[n] \leq N \bar{P}$, which is easy to find via using the bisection search [29].

3.2. Subproblem 2: Trajectory q Optimization. To make the optimization problem easier to handle, we first introduce the slack variable $\mathbf{C}=\left\{C[n]=\max _{k \in \mathscr{K}} R_{\mathrm{UE}_{k}}[n], n \in \mathscr{N}\right\}$; consequently, with given UAV transmit power $\mathbf{p}$, the original problem $(\mathrm{P} 1)$ can be transformed to be

(P3):

$\max _{\mathbf{q}, \mathbf{v}, \mathbf{a}, \mathbf{C}} \frac{\sum_{n=1}^{N}\left(B \log _{2}\left(1+\left(\gamma_{0} P[n] /\left(H^{2}+\|\mathbf{q}[n]\|^{2}\right)\right)\right)-C[n]\right)}{\sum_{n=1}^{N}\left(c_{1}\|\mathbf{v}[n]\|^{3}+\left(c_{2} /\|\mathbf{v}[n]\|\right)\left(1+\left(\|\mathbf{a}[n]\|^{2} / g^{2}\right)\right)\right)+\left(\Delta_{k} / \delta_{t}\right)}$, 


$$
\begin{gathered}
\text { s.t. } B \log _{2}\left(1+\frac{\gamma_{0} P[n]}{H^{2}+\left\|\mathbf{q}[n]-\mathbf{E}_{k}\right\|^{2}}\right) \leq C[n], \\
k \in \mathscr{K}, n \in \mathcal{N},
\end{gathered}
$$

However, (P3) is still intractable due to its fractional objective function with a nonconcave numerator and nonconvex constraint in (15b). In this case, by introducing slack variables $\quad \mathbf{u}=\{u[n]=\|\mathbf{v}[n]\|, n \in \mathscr{N}\} \quad$ and $\quad \mathbf{t}=\left\{t_{k}[n]=\right.$ $\left.\left\|\mathbf{q}[n]-\mathbf{E}_{k}\right\|^{2}+H^{2}, k \in \mathscr{K}, n \in \mathscr{N}\right\}$, (P3) can be reformulated as

(P3.1):

$\max _{\mathbf{q}, \mathbf{v}, \mathbf{a}, \mathbf{C}, \mathbf{t}, \mathbf{u}} \frac{\sum_{n=1}^{N}\left(B \log _{2}\left(1+\left(\gamma_{0} P[n] /\left(H^{2}+\|\mathbf{q}[n]\|^{2}\right)\right)\right)-C[n]\right)}{\sum_{n=1}^{N}\left(c_{1}\|\mathbf{v}[n]\|^{3}+\left(c_{2} / u[n]\right)+\left(c_{2}\|\mathbf{a}[n]\|^{2} / \mathbf{g}^{2} u[n]\right)\right)+\left(\Delta_{k} / \delta_{t}\right)}$,

$$
\begin{gathered}
\text { s.t.u[n] } \geq 0, n \in \mathcal{N}, \\
\|\mathbf{v}[n]\|^{2} \geq u^{2}[n], n \in \mathcal{N}, \\
\left\|\mathbf{q}[n]-\mathbf{E}_{k}\right\|^{2}+H^{2} \geq t_{k}[n], n \in \mathscr{N}, k \in \mathscr{K}, \\
B \log _{2}\left(1+\frac{\gamma_{0} P[n]}{\mathbf{t}}\right) \leq C[n], n \in \mathcal{N}, k \in \mathscr{K},
\end{gathered}
$$

It can be verified that constraints (16c) and (16d) should hold with equalities at the optimal solution of problem (P3.1), since otherwise $u[n]$ and $t_{k}[n]$ can be always increased to improve the objective value. Thus, problems (P3.1) and (P3) are equivalent. Nevertheless, problem (P3.1) is still a nonconvex function due to the nonconvexity of constraints (16c) and (16d). Hence, to tackle the nonconvex constraints, the SCA technique is leveraged to seek an efficient suboptimal solution. Specifically, since the terms $\|\mathbf{v}[n]\|^{2}$ and $\| \mathbf{q}[n]$ $-\mathbf{E}_{k} \|^{2}+H^{2}$ are, respectively, convex with regard to $\mathbf{v}[n]$ and $\mathbf{q}[n]$, their lower bound can be obtained via the firstorder Taylor expansion at any given local point $\left\{\mathbf{v}^{(r)}[n]\right\}$ and $\left\{\mathbf{q}^{(r)}[n]\right\}$ in the $r$ th iteration as follows:

$$
\begin{aligned}
& \|\mathbf{v}[n]\|^{2} \geq\left\|\mathbf{v}^{(r)}[n]\right\|^{2}+2\left(\mathbf{v}^{(r)}[n]\right)^{T}\left(\mathbf{v}[n]-\mathbf{v}^{(r)}[n]\right), n \in \mathcal{N}, \\
& \left\|\mathbf{q}[n]-\mathbf{E}_{k}\right\|^{2}+H^{2} \geq\left\|\mathbf{q}^{(r)}[n]-\mathbf{E}_{k}\right\|^{2}+H^{2} \\
& \quad+2\left(\left(\mathbf{q}^{(r)}[n]\right)^{T}-\mathbf{E}_{k}^{T}\right)\left(\mathbf{q}[n]-\mathbf{q}^{(r)}[n]\right), k \in \mathscr{K}, n \in \mathscr{N} .
\end{aligned}
$$

Similarly, to get to grips with the nonconvexity of the first-term $\log _{2}\left(1+\left(\gamma_{0} P[n] /\left(H^{2}+\|\mathbf{q}[n]\|^{2}\right)\right)\right)$ in the numerator of the objective function in (16a), by using the firstorder Taylor series approximation at any given $\left\{\mathbf{q}^{(r)}[n]\right\}$ over the $r$ th iteration, $\log _{2}\left(1+\left(\gamma_{0} P[n] /\left(H^{2}+\|\mathbf{q}[n]\|^{2}\right)\right)\right)$ can be lower bounded by

$$
\begin{gathered}
\log _{2}\left(1+\frac{\gamma_{0} P[n]}{H^{2}+\|\mathbf{q}[n]\|^{2}}\right) \geq \alpha_{j}[n]-\beta_{j}[n] \\
\cdot\left(\|\mathbf{q}[n]\|^{2}-\left\|\mathbf{q}^{(r)}[n]\right\|^{2}\right) \triangleq R_{\mathrm{lb}}[n],
\end{gathered}
$$

where

$$
\begin{gathered}
\alpha_{j}[n]=\log _{2}\left(1+\frac{\gamma_{0} P[n]}{H^{2}+\left\|\mathbf{q}^{(r)}[n]\right\|^{2}}\right), \\
\beta_{j}[n]=\frac{\gamma_{0} P[n]}{\left(H^{2}+\left\|\mathbf{q}^{(r)}[n]\right\|^{2}+\gamma_{0} p[n]\right)\left(H^{2}+\left\|\mathbf{q}^{(r)}[n]\right\|^{2}\right) \ln 2} .
\end{gathered}
$$

With (17)-(19), for any given local point $\left\{\mathbf{q}^{(r)}[n]\right\}$ and $\left\{\mathbf{v}^{(r)}[n]\right\}$, we can obtain an approximate convex problem for (P3.1), i.e.,

\section{(P3.2):}

$\max _{\mathbf{q}, \mathbf{v}, \mathbf{a}, \mathbf{C}, \mathbf{t}, \mathbf{u}} \frac{\sum_{n=1}^{N}\left(B R_{\mathrm{lb}}[n]-C[n]\right)}{\sum_{n=1}^{N}\left(c_{1}\|\mathbf{v}[n]\|^{3}+\left(c_{2} / u[n]\right)+\left(c_{2}\|\mathbf{a}[n]\|^{2} / \mathbf{g}^{2} u[n]\right)\right)+\left(\Delta_{k} / \delta_{t}\right)}$,
s.t.(1), (16b), (16e), (17), (18).

Now, the numerator and denominator of the objective function of problem (P3.2) are concave and convex, respectively, and the feasible region of problem (P3.2) is also convex. Hence, the fractional maximization problem of (P3.2) can be efficiently solved by Dinkelbach's algorithm [30]. Specifically, we define $f(\mathbf{q}, \mathbf{C}, \mathbf{t})=\sum_{n=1}^{N}\left(B R_{\mathrm{lb}}[n]-C[n]\right)$ and $\mathrm{g}(\mathbf{v}, \mathbf{a}, \mathbf{u})=\sum_{n=1}^{N}\left(c_{1}\|\mathbf{v}[n]\|^{3}+\left(c_{2} / u[n]\right)+\left(c_{2}\|\mathbf{a}[n]\|^{2} / \mathbf{g}^{2} u[n]\right)\right)$ $+\left(\Delta_{k} / \delta_{t}\right)$, and let $\mu^{*}$ be the maximum SEE of problem (P3.2), which can be expressed as

$$
\mu^{*}=\frac{f\left(\mathbf{q}^{*}, \mathbf{C}^{*}, \mathbf{t}^{*}\right)}{\mathrm{g}\left(\mathbf{v}^{*}, \mathbf{a}^{*}, \mathbf{u}^{*}\right)}=\max _{\mathbf{q}, \mathbf{C}, \mathbf{v}, \mathbf{a}, \mathbf{t}, \mathbf{u}} \frac{f(\mathbf{q}, \mathbf{C}, \mathbf{t})}{\mathrm{g}(\mathbf{v}, \mathbf{a}, \mathbf{u})} .
$$

According to [30], the optimal feasible solution of problem (P3.2) is achieved (i.e., the maximum value of SEE $\mu^{*}$ ) if and only if

$$
\begin{gathered}
\max _{\mathbf{q}, \mathbf{C}, \mathbf{v}, \mathbf{a}, \mathbf{t}, \mathbf{u}}\left[f(\mathbf{q}, \mathbf{C}, \mathbf{t})-\mu^{*} \mathrm{~g}(\mathbf{v}, \mathbf{a}, \mathbf{u})\right]=f\left(\mathbf{q}^{*}, \mathbf{C}^{*}, \mathbf{t}^{*}\right) \\
-\mu^{*} \mathrm{~g}\left(\mathbf{v}^{*}, \mathbf{a}^{*}, \mathbf{u}^{*}\right)=0 .
\end{gathered}
$$

Hence, the fractional objective function of problem (P3.2) is transformed into the following equivalent problem:

$$
\begin{gathered}
\text { (P3.3): } F(\mu)=\max _{\mathbf{q}, \mathbf{C}, \mathbf{v}, \mathbf{a}, \mathbf{u}, \mathbf{u}}[f(\mathbf{q}, \mathbf{C}, \mathbf{t})-\mu \mathrm{g}(\mathbf{v}, \mathbf{a}, \mathbf{u})], \\
\text { s.t.(1), (16b), (16e), (17), (18). }
\end{gathered}
$$




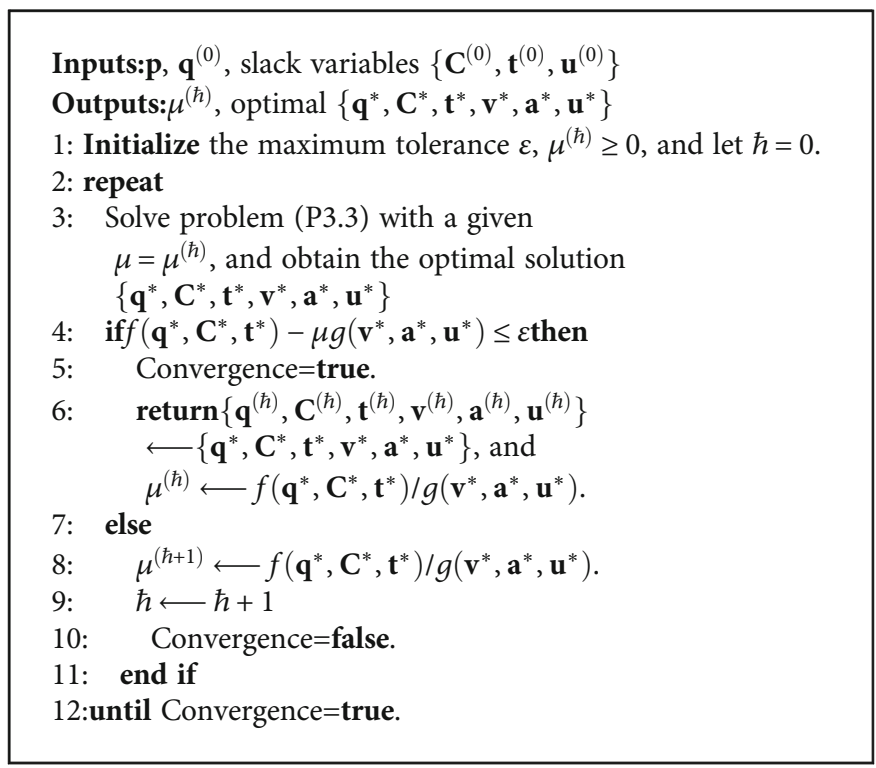

Algorithm 1: Proposed Dinkelbach's algorithm for problem (P3.2).

Now, (P3.3) is convex and can be solved effectively via classical tools, e.g., CVX [31]. Note that an iterative updating for $\mu$ is required to find the root of $F(\mu)=0$. Therefore, we can solve (P3.2) via optimizing the UAV's trajectory in (P3.3) iteratively, where the details on the stages of problem (P3.2) solving by applying Dinkelbach's algorithm are presented in Algorithm 1. It should be noted that leveraging Dinkelbach's algorithm to solve (P3.2) can guarantee the global optimality.

3.3. Overall Algorithm. In a nutshell, an iterative algorithm for solving the original problem (P1) by leveraging the block coordinate descent method and Dinkelbach's algorithm is proposed as summarized in Algorithm 2. Next, the convergence of the proposed Algorithm 2 is analyzed as follows. Since (P2) is optimally solved, we have $\eta\left(\mathbf{p}^{(r)}, \mathbf{q}^{(r)}\right) \leq \eta($ $\left.\mathbf{p}^{(r+1)}, \mathbf{q}^{(r)}\right)={ }^{(a)} \eta_{\mathrm{lb}}\left(\mathbf{p}^{(r+1)}, \mathbf{q}^{(r)}\right) \leq^{(b)} \eta_{\mathrm{lb}}\left(\mathbf{p}^{(r+1)}, \mathbf{q}^{(r+1)}\right) \leq^{(c)} \eta($ $\mathbf{p}^{(r+1)}, \mathbf{q}^{(r+1)}$, where $(a)$ holds because the Taylor expansions in (17)-(19) are tight at given $\mathbf{q}^{(r)},(b)$ comes from the fact that the optimal solution of (P3.2) is attained optimally via Dinkelbach's method, and $(c)$ is true since the maximal SEE of (P3.2) is a lower bound of that of (P3.1). Therefore, in each iteration of algorithm 2 , the $\eta\left(\mathbf{p}^{(r)}, \mathbf{q}^{(r)}\right)$ obtained is nondecreasing .

3.4. Competitive Schemes. In this subsection, to obtain more performance insights, we consider the following two benchmark schemes.

3.4.1. Secrecy Rate Maximization (SRM). For this scheme, only the secrecy rate is maximized by jointly optimizing the UAV's transmit power $\mathbf{p}$ and UAV's trajectory $\mathbf{q}$, where the corresponding optimization problem can be formulated as follows:

$$
\begin{gathered}
(\mathrm{P} 4): \max _{\mathbf{p}, \mathbf{q}} \sum_{n=1}^{N}\left[R_{\mathrm{UB}}[n]-\max _{k \in \mathscr{K}} R_{\mathrm{UE}_{k}}[n]\right], \\
\text { s.t.(1), (4). }
\end{gathered}
$$

Although problem (P4) is challenging to solve optimally owing to its nonconvex nature, similar as [32], an alternating iteration algorithm can be leveraged to effectively solve this problem.

3.4.2. Constrained Energy Minimization (CEM). For this scheme, the total UAV's energy consumption minimization via optimizing the UAV trajectory $\mathbf{q}$ is considered when the requirement communication performance is satisfied. Hence, we formulate the energy-minimization problem with secrecy rate constrained as

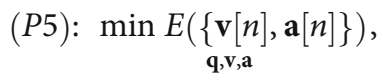

$$
\text { s.t. } \frac{\delta_{t}}{N B} \sum_{n=1}^{N}\left(R_{\mathrm{UB}}[n]-\max _{k \in \mathscr{K}} R_{\mathrm{UE}_{k}}[n]\right) \geq 0.5 \text {, }
$$

$$
\text { (1), (4). }
$$

Although (P5) is also nonconvex, by introducing the slack variable $\mathbf{t}$, (P5) can be transformed into a series of convex subproblems by means of the SCA approach, which can be then iteratively solved using CVX. The details are omitted for simplicity. 


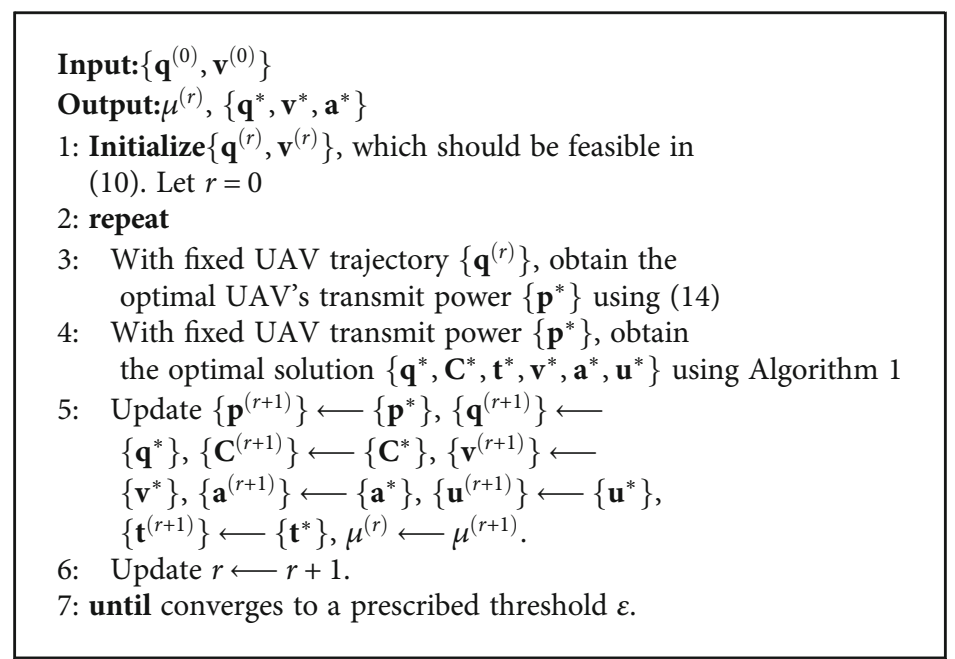

Algorithm 2: Proposed iterative algorithm to solve (P1).

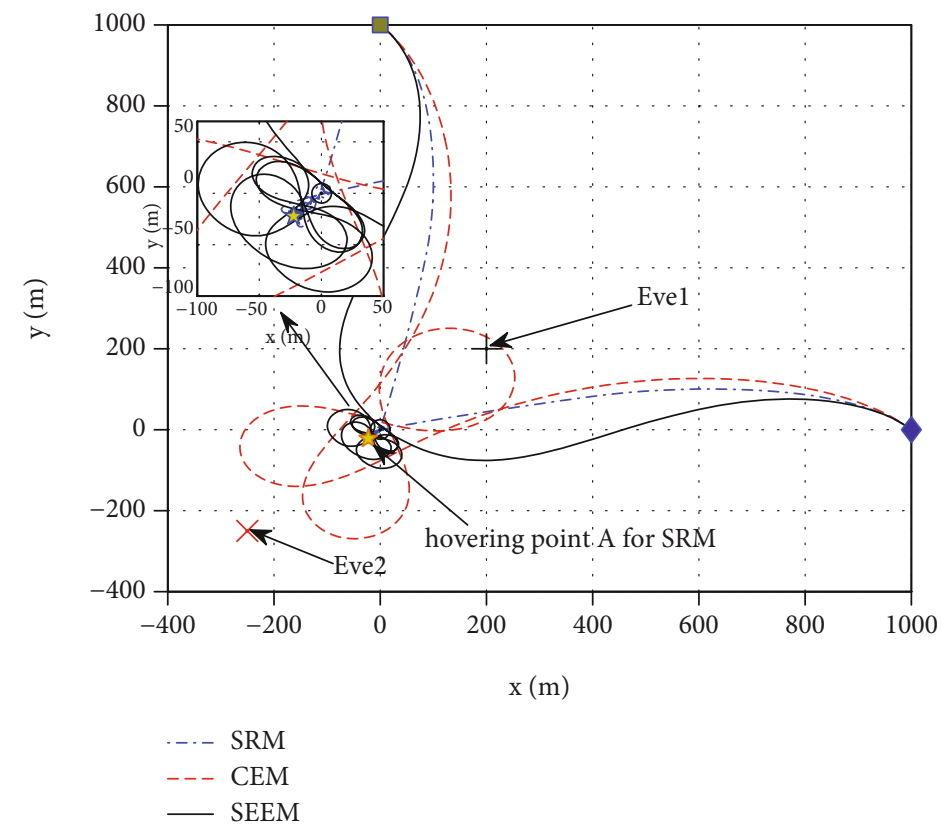

Figure 2: UAV's trajectories for different schemes.

\section{Simulation Results}

In this section, numerical results are presented to validate the performance of our proposed SEE maximization (SEEM) algorithm. In the simulation, the noise power spectrum density and the total communication bandwidth are $N_{0}=-170$ $\mathrm{dBm} / \mathrm{Hz}$ and $B=1 \mathrm{MHz}$, respectively. The average transmit power budget of the UAV is set as $\bar{P}=10 \mathrm{dBm}$ and the peak transmit power limit is to $P_{\text {peak }}=4 \bar{P}$. The reference SNR at distance $d_{0}=1 \mathrm{~m}$ is $\gamma_{0}=70 \mathrm{~dB}$. Bob's location is fixed at $[0,0]^{T}$, and the UAV's start and end points are, respectively, set to $\mathbf{q}_{0}=[0,1000]^{T}$ and $\mathbf{q}_{\mathrm{F}}=[1000,0]^{T}$. There are $K=2$ Eves, whose locations are set to $\mathbf{E}_{1}=[200,200]^{T}$ and $\mathbf{E}_{2}=$ $[-250,-250]^{T}$, respectively. Moreover, we set $T=160 \mathrm{~s}, \delta_{t}$ $=0.5 \mathrm{~s}, V_{\min }=4 \mathrm{~m} / \mathrm{s}, V_{\max }=100 \mathrm{~m} / \mathrm{s}, a_{\max }=5 \mathrm{~m} / \mathrm{s}^{2}, \varepsilon=1$
TABLE 1: SEE comparison of various schemes at $T=160 \mathrm{~s}$.

\begin{tabular}{lccccc}
\hline & $\begin{array}{c}\text { Average } \\
\text { speed } \\
(\mathrm{m} / \mathrm{s})\end{array}$ & $\begin{array}{c}\text { Average } \\
\text { acceleration } \\
\left(\mathrm{m}^{2} / \mathrm{s}\right)\end{array}$ & $\begin{array}{c}\text { Average } \\
\text { secrecy } \\
\text { rate } \\
(\mathrm{Mbps})\end{array}$ & $\begin{array}{c}\text { Average } \\
\text { power } \\
\text { (Watts) }\end{array}$ & $\begin{array}{c}\text { Secrecy } \\
\text { energy } \\
\text { efficiency } \\
\text { (kbits/ } \\
\text { Joule })\end{array}$ \\
\hline SSRM & 16.12 & 4.77 & 2.49 & 511.11 & 4.87 \\
CCEM & 26.98 & 2.60 & 0.64 & 112.48 & 5.73 \\
SEEM & 21.78 & 4.30 & 2.15 & 188.24 & 11.43 \\
\hline
\end{tabular}

$0^{-4}, \quad c_{1}=9.26 \times 10^{-4}, \quad c_{2}=2250$, and $\mathbf{v}_{0}=\mathbf{v}_{\mathrm{F}}=30 \overleftarrow{\mathbf{v}}_{0 \mathrm{~F}}$ with $\overleftarrow{\mathbf{v}}_{0 \mathrm{~F}}=\left(\mathbf{q}_{\mathrm{F}}-\mathbf{q}_{0}\right) /\left\|\mathbf{q}_{\mathrm{F}}-\mathbf{q}_{0}\right\|^{2}$ denoting the direction from $\mathbf{q}_{0}$ to $\mathbf{q}_{\mathrm{F}}$. 


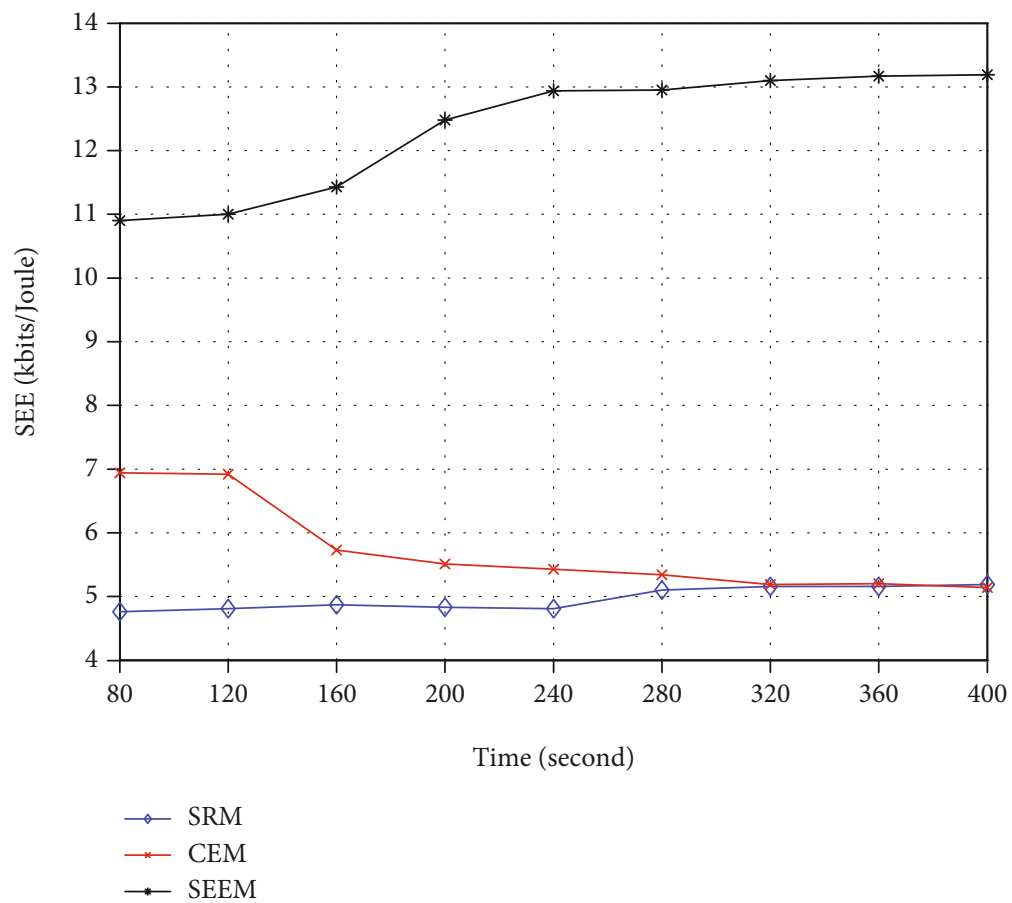

Figure 3: SEE of various schemes versus $T$.

Figure 2 shows the designed trajectories obtained from different schemes. The locations of Bob, $E_{1}, E_{2}$, and the start and end points of UAV, are separately marked with $O,+, \times$, $\square$ and $\diamond$, respectively. From Figure 2, one can observe that all the trajectories by the proposed SEEM and two benchmarks schemes show significant difference. For the SRM scheme, UAV first flies along a relatively straight route with the maximum acceleration to arrive at the point $\mathbf{A}=$ $[-22.23,-22.23,100]^{T}$ in the bottom left of Bob and then hovers around $\mathbf{A}$ as long as possible, finally moves towards $\mathbf{q}_{\mathrm{F}}$ with the maximum acceleration. The hovering point $\mathbf{A}$ strikes a balance between improving the destination link and weakening the eavesdropper link, thus achieving the maximum secrecy rate. For the CEM scheme, different from the large "U"-shaped path in [20], due to the constraint of the imposed secrecy rate, the UAV follows a path with a large and smooth turning radius, like approximately a big " 8 "-shaped path. Note that the UAV hovers around longer on the top right of $\mathbf{E}_{2}$ than on the bottom left of $\mathbf{E}_{1}$ since $\mathbf{E}_{2}$ is farther from Bob than $\mathbf{E}_{1}$. Such trajectory is less power consuming while meeting the communication performance requirements. Consequently, the proposed SEEM scheme, upon approaching point $\mathbf{A}$, yields a trajectory with an approximately small " 8 "-shaped in order to provide adequately secure communication channel but not consume excessive power.

Table 1 provides the detailed system performance comparison for three schemes. It is observed from Table 1 that the SRM scheme can obtain the largest average secrecy rate but with the highest power consumption. On the contrary, CEM scheme consumes the lowest possible energy but also achieves the smallest average secrecy rate. Thus, both bench- mark schemes are energy inefficient as expected. Only the proposed SEEM scheme can obtain a good balance between the achievable average secrecy rate and power consumption, thus achieving a significantly higher SEE than two benchmark schemes.

Figure 3 shows the SEE of different schemes versus flight period $T$. It is observed that for the SEEM scheme and the SRM scheme, the SEE increases with a larger flight period $T$ at first then tends to be stable, while for the CEM scheme, it initially decreases with $T$ before remaining the same stable SEE as for the SRM scheme. This is because when the UAV hovers around point A with a small " 8 "-shaped path, the SEE gain is higher. Therefore, for the SEEM scheme as flight period $T$ increases, the hovering time of UAV around $\mathbf{A}$ accounts for a larger proportion of the entire flight period $T$ until approaching 1; thus, the SEE increases until it stabilizes around $13.19 \mathrm{kbits} /$ Joule. Nevertheless, for the SRM scheme, since the UAV is energy inefficiently hovering over point $\mathbf{A}$, the SEE gain is limited with $T$. In particular, for the CEM scheme, when $T$ increases, the UAV will fly and hover at a larger radius to minimize the energy consumption, thus leading to the decrease of SEE until the stable value $5.14 \mathrm{kbits} /$ Joule, the same SEE as for the SRM scheme. Obviously, for any flight period $T$, our proposed scheme achieves the highest SEE, which validates the superiority of the SEEM scheme.

\section{Conclusion}

In this paper, we have scrutinized the energy-efficient UAVground secure transmission system. Specifically, we have purposed to maximize the worst-case SEE via the UAV's 
trajectory design and power control. However, the SEE maximization problem is challenging to solve optimally because of its inherent nonconvexity. Therefore, an effective iterative algorithm is proposed by leveraging block coordinate descent method and Dinkelbach's algorithm as well as the SCA technique to maximize the system SEE. Numerical results demonstrate that our proposed SEEM scheme achieves a significantly higher system SEE than the benchmark schemes.

\section{Data Availability}

The data used to support the findings of this study are available from the corresponding author upon request.

\section{Conflicts of Interest}

The authors declare no conflict of interest.

\section{Acknowledgments}

This work was partly supported by the Foundation for Distinguished Young Talents Training Programme of Jiangxi (No. 20171BCB23006), Key Research and Development Project of Jiangxi Province (No. 20202BBE53019), 03 Special Funds of Jiangxi Province (Nos. 20204ABC03A05 and 20204ABC03A37).

\section{References}

[1] Unmanned aerial vehicle (UAV) market by component (hardware, software), class (mini UAVs, micro UAVs), end user (military, commercial, agriculture), type (fixed wing, rotary-wing UAVs), capacity, and mode of operation-global forecast to 2027, 2020, https://www.giiresearch.com/report/meti942389unmanned-aerial-vehicle-uav-market-bycomponent.html.

[2] Y. ZENG, R. ZHANG, and T. J. LIM, "Wireless communications with unmanned aerial vehicles: opportunities and challenges," IEEE Communications Magazine, vol. 54, no. 5, pp. 36-42, 2016.

[3] J. Lyu, Y. Zeng, R. Zhang, and T. J. Lim, "Placement optimization of uav-mounted mobile base stations," IEEE Communications Letters, vol. 21, no. 3, pp. 604-607, 2016.

[4] S. Zhu, L. Gui, N. Cheng, F. Sun, and Q. Zhang, "Joint design of access point selection and path planning for uav-assisted cellular networks," IEEE Internet of Things Journal, vol. 7, no. 1, pp. 220-233, 2019.

[5] J.-H. Lee, K.-H. Park, Y.-C. Ko, and M.-S. Alouini, “Throughput maximization of mixed FSO/RF UAV-aided mobile relaying with a buffer," IEEE Transactions onWireless Communications, vol. 20, no. 1, pp. 683-694, 2021.

[6] H. Hu, K. Xiong, G. Qu, Q. Ni, P. Fan, and K. B. Letaief, “AoIminimal trajectory planning and data collection in UAVassisted wireless powered IoT networks," IEEE Internet of Things Journal, vol. 8, no. 2, pp. 1211-1223, 2021.

[7] A. Li, Q. Wu, and R. Zhang, "UAV-enabled cooperative jamming for improving secrecy of ground wiretap channel," IEEE Wireless Communications Letters, vol. 8, no. 1, pp. 181-184, 2019.
[8] Y. Zhou, C. Pan, P. L. Yeoh et al., "Secure communications for UAV-enabled mobile edge computing systems," IEEE Transactions on Communications, vol. 68, no. 1, pp. 376-388, 2020.

[9] W. WANG, K. C. TEH, and LI K H, "Generalized relay selection for improved security in cooperative DF relay networks," IEEE Wireless Communications Letters, vol. 5, no. 1, pp. 28-31, 2015.

[10] X. Chen, D. W. K. Ng, W. H. Gerstacker, and H.-H. Chen, “A survey on multiple-antenna techniques for physical layer security," IEEE Communications Surveys \& Tutorials, vol. 19, no. 2, pp. 1027-1053, 2016.

[11] I. BANG, S. M. KIM, and D. K. SUNG, "Artificial noise-aided user scheduling for optimal secrecy multiuser diversity," IEEE Communications Letters, vol. 21, no. 3, pp. 528-531, 2016.

[12] H. Hui, A. L. Swindlehurst, G. Li, and J. Liang, "Secure relay and jammer selection for physical layer security," IEEE Signal Processing Letters, vol. 22, no. 8, pp. 1147-1151, 2015.

[13] K. Cumanan, G. C. Alexandropoulos, Z. Ding, and G. K. Karagiannidis, "Secure communications with cooperative jamming: optimal power allocation and secrecy outage analysis," IEEE Transactions on Vehicular Technology, vol. 66, no. 8, pp. 7495-7505, 2017.

[14] M. Caris, S. Stanko, M. Malanowski et al., "mm-Wave SAR demonstrator as a test bed for advanced solutions in microwave imaging," IEEE Aerospace and Electronic Systems Magazine, vol. 29, no. 7, pp. 8-15, 2014.

[15] G. Zhang, Q. Wu, M. Cui, and R. Zhang, "Securing UAV communications via joint trajectory and power control," IEEE Transactions on Wireless Communications, vol. 18, no. 2, pp. 1376-1389, 2019.

[16] A. Li and W. Zhang, "Mobile jammer-aided secure UAV communications via trajectory design and power control," China Communications, vol. 15, no. 8, pp. 141-151, 2018.

[17] Y. Cai, F. Cui, Q. Shi, M. Zhao, and G. Y. Li, "Dual-UAVenabled secure communications: joint trajectory design and user scheduling," IEEE Journal on Selected Areas in Communications, vol. 36, no. 9, pp. 1972-1985, 2018.

[18] H. Lee, S. Eom, J. Park, and I. Lee, "UAV-aided secure communications with cooperative jamming," IEEE Transactions on Vehicular Technology, vol. 67, no. 10, pp. 9385-9392, 2018.

[19] C. ZHONG, J. YAO, and J. XU, "Secure UAV communication with cooperative jamming and trajectory control," IEEE Communications Letters, vol. 23, no. 2, pp. 286-289, 2018.

[20] Y. ZENG and R. ZHANG, "Energy-efficient UAV communication with trajectory optimization," IEEE Transactions on Wireless Communications, vol. 16, no. 6, pp. 3747-3760, 2017.

[21] S. AHMED, M. Z. CHOWDHURY, and Y. M. JANG, "Energyefficient UAV relaying communications to serve ground nodes," IEEE Communications Letters, vol. 24, no. 4, pp. 849-852, 2020.

[22] F. Zeng, Z. Hu, Z. Xiao et al., "Resource allocation and trajectory optimization for QoE provisioning in energy-efficient UAV-enabled wireless networks," IEEE Transactions on Vehicular Technology, vol. 69, no. 7, pp. 7634-7647, 2020.

[23] S. Seng, G. Yang, X. Li, H. Ji, and C. Luo, "Energy efficient communications in unmanned aerial relaying systems," IEEE Transactions on Network Science and Engineering, 2021.

[24] L. Xiao, Y. Xu, D. Yang, and Y. Zeng, "Secrecy energy efficiency maximization for UAV-enabled mobile relaying," IEEE Transactions on Green Communications and Networking, vol. 4, no. 1, pp. 180-193, 2019. 
[25] B. Duo, Q. Wu, X. Yuan, and R. Zhang, "Energy efficiency maximization for full-duplex UAV secrecy communication," IEEE Transactions on Vehicular Technology, vol. 69, no. 4, pp. 4590-4595, 2020.

[26] C. Stöcker, R. Bennett, F. Nex, M. Gerke, and J. Zevenbergen, "Review of the current state of UAV regulations," Remote Sensing, vol. 9, no. 5, 2017.

[27] X. Lin, V. Yajnanarayana, S. D. Muruganathan et al., "The sky is not the limit: LTE for unmanned aerial vehicles," IEEE Communications Magazine, vol. 56, no. 4, pp. 204-210, 2018.

[28] P. K. Gopala, L. Lai, and H. El Gamal, "On the secrecy capacity of fading channels," IEEE Transactions on Information Theory, vol. 54, no. 10, pp. 4687-4698, 2008.

[29] S. BOYD, L. VANDENBERGHE, and L. FAYBUSOVICH, "Convex optimization," IEEE Transactions on Automatic Control, vol. 51, no. 11, pp. 1859-1859, 2006.

[30] J.-P. Crouzeix and J. A. Ferland, "Algorithms for generalized fractional programming," Mathematical Programming, vol. 52, no. 1-3, pp. 191-207, 1991.

[31] M. GRANT, S. BOYD, and Y. YE, CVX: Matlab software for disciplined convex programming, 2008, http://cvxr.com/cvx.

[32] M. Cui, G. Zhang, Q. Wu, and D. W. K. Ng, "Robust trajectory and transmit power design for secure UAV communications," IEEE Transactions on Vehicular Technology, vol. 67, no. 9, pp. 9042-9046, 2018. 